Article

\title{
Statistical Hauser-Feshbach Model Description of $(n, \alpha)$ Reaction Cross Sections for the Weak s-Process
}

\author{
Sema Küçüksucu ${ }^{1,2}$ (D), Mustafa Yiğit ${ }^{2}$ and Nils Paar ${ }^{1, *(D)}$ \\ 1 Department of Physics, Faculty of Science, University of Zagreb, Bijenička c. 32, 10000 Zagreb, Croatia; \\ semak@phy.hr \\ 2 Department of Physics, Faculty of Arts and Science, Aksaray University, 68100 Aksaray, Turkey; \\ mustafayigit@aksaray.edu.tr \\ * Correspondence: npaar@phy.hr
}

Citation: Küçüksucu, S.; Yiğit, M.; Paar, N. Statistical Hauser-Feshbach Model Description of $(n, \alpha)$ Reaction Cross Sections for the Weak s-Process Universe 2022, 8, 25. https://doi.org/ $10.3390 /$ universe 8010025

Academic Editors: David Blaschke, Konstantin Maslov, Elena Litvinova and Evgeni Kolomeitsev

Received: 29 November 2021

Accepted: 30 December 2021

Published: 1 January 2022

Publisher's Note: MDPI stays neutral with regard to jurisdictional claims in published maps and institutional affiliations.

Copyright: (c) 2022 by the authors. Licensee MDPI, Basel, Switzerland. This article is an open access article distributed under the terms and conditions of the Creative Commons Attribution (CC BY) license (https:// creativecommons.org/licenses/by/ $4.0 /)$.

\begin{abstract}
The $(n, \alpha)$ reaction contributes in many processes of energy generation and nucleosynthesis in stellar environment. Since experimental data are available for a limited number of nuclei and in restricted energy ranges, at present only theoretical studies can provide predictions for all astrophysically relevant $(n, \alpha)$ reaction cross sections. The purpose of this work is to study $(n, \alpha)$ reaction cross sections for a set of nuclei contributing in the weak s-process nucleosynthesis. Theory framework is based on the statistical Hauser-Feshbach model implemented in TALYS code with nuclear masses and level densities based on Skyrme energy density functional. In addition to the analysis of the properties of calculated $(n, \alpha)$ cross sections, the Maxwellian averaged cross sections are described and analyzed for the range of temperatures in stellar environment. Model calculations determined astrophysically relevant energy windows in which $(n, \alpha)$ reactions occur in stars. In order to reduce the uncertainties in modeling $(n, \alpha)$ reaction cross sections for the s-process, novel experimental studies are called for. Presented results on the effective energy windows for $(n, \alpha)$ reaction in weak s-process provide a guidance for the priority energy ranges in the future experimental studies.
\end{abstract}

Keywords: neutron induced reactions; alpha emission; Hauser-Feshbach model; s-process

\section{Introduction}

Fascinating phenomena in the universe, such as evolution of stars, supernova explosions and neutron-star mergers, as well as the synthesis of chemical elements that occur in stellar environment, crucially depend on a variety of nuclear reactions [1-4]. Nuclear reaction cross sections and rates are an essential for understanding the elemental abundances in our solar system and the galaxy. Since the properties of many nuclear reactions and involved nuclei in stellar environment still remain beyond the reach of the most advanced experimental facilities, theoretical modeling is necessary to provide relevant cross sections for astrophysical applications $[4,5]$. Available nuclear reaction cross sections, both from the experiment and model calculations are summarized in various data bases [6-12].

The stellar burning phases of stars build chemical elements up to the Iron group by sequences of fusion reactions [5]. However, elements heavier than Iron are produced by other types or nuclear processes, in different stellar environment [4]. Of particular importance are neutron capture reactions that govern two key processes in nucleosynthesis: (i) slow neutron capture process, known as s-process, which takes place e.g., in giant stars $[13,14]$ and rotating massive metal poor stars [15], and (ii) rapid neutron capture process, known as r-process which occurs in explosive stellar environments such as supernova [16-19] and neutron star mergers [20-23]. The description of neutron capture reactions crucially depends on the nuclear structure and excitation properties of target and daughter nuclei involved [24]. One of the key quantities is the reaction $Q$ value, which in general can have both positive and negative values, and the reaction outcome is governed by the energy of the incoming neutrons, i.e., by the respective astrophysical conditions which determine 
the neutron energy distribution. Advanced theoretical modeling of nuclear properties and respective neutron induced reactions is essential to provide reliable input for astrophysical applications [4].

The focus of this study is the $(n, \alpha)$ reaction in nuclei of relevance for the s-process nucleosynthesis [25]. For example, ${ }^{41} \mathrm{Ca}(n, \alpha){ }^{38} \mathrm{Ar}$ is considered as the most important neutron-induced reaction on ${ }^{41} \mathrm{Ca}$ in stellar conditions of the weak s-process at temperature about $300 \mathrm{MK}$ [26], i.e., it strongly dominates over the neutron induced $\gamma$-emission [27]. The $(n, \alpha)$ reaction contributes in many processes of energy generation and nucleosynthesis. It is also an important reaction in primordial and stellar CNO cycle [28]. In order to determine reaction rates in stellar environment, the reaction cross section as a function of neutron energy is an essential ingredient [29]. Although the experimental studies provide some of the necessary cross sections, for systematical implementation of $(n, \alpha)$ reactions in nucleosynthesis calculations theoretical modeling of the cross sections is indispensable. As pointed out in Ref. [30], limited amount of experimental data for $(n, \alpha)$ reaction in the $1 \mathrm{keV}-1 \mathrm{MeV}$ region, restricts comprehensive data analysis. Only small changes in the neutron capture reaction cross sections could have significant implications on the path of the nuclear processes that govern the synthesis of chemical elements [31]. Therefore, it is necessary to investigate $(n, \alpha)$ cross sections from various theoretical models, with different assumptions and microscopic nuclear properties in order to provide complementary description that could also have implications on the s-process network calculations [25].

The experimental data on $(n, \alpha)$ reaction cross sections for the s-process are available for a limited set of nuclei and in restricted ranges of neutron energies [6,32,33]. Recent measurements of $(n, \alpha)$ reactions include e.g., refs. [26,34-43]. The $(n, \alpha)$ reaction has been measured in the s-process branching point at ${ }^{59} \mathrm{Ni}$, in view of considerable differences between model predictions and experimental data [38,44]. Novel experimental techniques and detector systems have been recently developed to provide accurate new data $[45,46]$. The NICE-detector opened new perspectives to determine neutron capture cross-sections with charged particle in the exit channel with sufficient accuracy, for different nuclear and astrophysical applications [47]. Presently available experimental data and the corresponding empirical formulae cannot provide complete cross sections necessary for applications in astrophysical and nucleosynthesis models. Therefore, in this work we aim to investigate $(n, \alpha)$ reaction cross sections from the theory side, based on Hauser-Feshbach statistical model $[48,49]$ implemented in the nuclear reaction program TALYS-1.95 [50,51]. When possible, nuclear properties needed for this study are based on the energy density functional theory, using Skyrme-type functional with improved description of pairing, HFB-17 [52]. Due to its relevance in the s-process nucleosynthesis, the $(n, \alpha)$ reactions will also be analyzed for the range of temperatures characteristic in stellar environment, by averaging the cross sections over the Maxwell-Boltzmann distribution.

The objective of this work is to study $(n, \alpha)$ reaction cross sections for nuclei introduced in the weak s-process network calculations of a massive star, including convective He burning core and shell C- burning [25]. The weak s-process produces most of the sprocess isotopes between iron and strontium [25]. As pointed out in Ref. [25], the s-process nucleosynthesis is rather uncertain, especially in the $C$ shell, due to uncertainties in the neutron capture cross sections. Therefore, in this study we aim to improve the knowledge on $(n, \alpha)$ reaction cross sections of importance for the weak s-process, with focus on twelve relevant nuclei as introduced in Ref. [25]. These nuclei are: ${ }^{17} \mathrm{O},{ }^{18} \mathrm{~F},{ }^{22} \mathrm{Na},{ }^{26} \mathrm{Al},{ }^{33} \mathrm{~S},{ }^{37} \mathrm{Ar}$, ${ }^{39} \mathrm{Ar},{ }^{40} \mathrm{~K},{ }^{41} \mathrm{Ca},{ }^{59} \mathrm{Ni},{ }^{65} \mathrm{Zn}$, and ${ }^{71} \mathrm{Ge}$. The reaction outcome depends on the respective $\mathrm{Q}$-values, that are listed for this set of nuclei in Table 1. For all nuclei the $\mathrm{Q}$ values are based on Skyrme HFB-17 interaction [52], except for ${ }^{17} \mathrm{O},{ }^{18} \mathrm{~F}$, and ${ }^{22} \mathrm{Na}$, where the experimental values $[53,54]$ are adopted since the model calculations resulted in larger differences when compared to the experiment (we use these values also in the forthcoming calculations of the cross sections). For all nuclei under consideration, the $Q$ values are positive, thus allowing $(n, \alpha)$ reaction already at low neutron energies, that is of particular relevance for the respective reaction rates in stellar environment, as will be discussed in Section 3.2. 
Table 1. Q-values for $(n, \alpha)$ reactions used in this work, obtained from Skyrme HFB-17 interaction [52], except for nuclei denoted with * where the experimental Q-values are adopted $[53,54]$.

\begin{tabular}{cc}
\hline Nucleus & $\mathbf{Q}(\mathbf{M e V})$ \\
\hline${ }^{17} \mathrm{O}$ & $1.817^{*}$ \\
${ }^{18} \mathrm{~F}$ & $6.418^{*}$ \\
${ }^{22} \mathrm{Na}$ & $1.952^{*}$ \\
${ }^{26} \mathrm{Al}$ & 2.462 \\
${ }^{33} \mathrm{~S}$ & 3.870 \\
${ }^{37} \mathrm{Ar}$ & 3.264 \\
${ }^{39} \mathrm{Ar}$ & 3.060 \\
${ }^{40} \mathrm{~K}$ & 2.973 \\
${ }^{41} \mathrm{Ca}$ & 6.101 \\
${ }^{59} \mathrm{Ni}$ & 5.711 \\
${ }^{65} \mathrm{Zn}$ & 6.223 \\
${ }^{71} \mathrm{Ge}$ & 5.704 \\
\hline
\end{tabular}

The Gamow peak represents one of the most important concepts in the study of thermonuclear reactions in stars [55-57]. The corresponding energy range known as Gamow window, determines the effective stellar energy region in which most chargedparticle induced nuclear reactions occur [55,56,58,59]. In Ref. [58] the Gamow windows of a variety of astrophysical reaction rates have been explored in a systematic study over the nuclide map using Hauser-Feshbach model. Since available experimental data on $(n, \alpha)$ reaction cross sections for s-process nuclei are incomplete, predictions of astrophysically relevant energy ranges are of importance for the future design and planning of nuclear astrophysics measurements, including those using radioactive ion beams at novel facilities. Therefore, one of the objectives of this work is to determine the astrophysically relevant energy window where the $(n, \alpha)$ reactions occur in stellar environment. In this way, we aim to provide the guidelines for the energy ranges to be measured in future experiments on s-process nuclei, that are necessary to reduce currently existing systematic uncertainties in model calculations.

The paper is organized as follows. Section 2 includes a brief overview of the theory framework and methods for $(n, \alpha)$ reactions and their implementation in TALYS code. In Section 3.1 the results on the cross sections for the s-process nuclei are presented and discussed. The results for astrophysically relevant energy ranges for $(n, \alpha)$ reactions in s-process nuclei and respective Maxwellian averaged cross sections are also given and discussed in Sections 3.2 and 3.3. Summary and conclusions are given in Section 4.

\section{Theory Framework}

Neutron induced reaction cross sections in this work are described using the statistical Hauser-Feshbach model implemented in the nuclear reaction program TALYS1.95 [50,51]. More details about the formalism of Hauser-Feshbach statistical model are given in refs. $[8,48,49]$. The model assumes the validity of the compound nucleus reaction mechanism and a statistical distribution of nuclear excited states [14,49]. This method is appropriate when the level density in the contributing energy window around the peak of the projectile energy distribution is sufficiently high to justify a statistical treatment [8]. The compound nucleus formation dominates when the energy of the incident particle is low enough, below $\approx 20 \mathrm{MeV}$. This condition is almost always satisfied in astrophysical environments. Among various outgoing particles obtained from the compound nucleus, dominating contributions to the cross section come from neutron, $\gamma$-ray, proton, and $\alpha$ particle. Comprehensive Hauser-Feshbach model calculations of astrophysical reaction rates are available in ref. [8].

In the present work, nuclear reaction code TALYS-1.95 is used to calculate $(n, \alpha)$ reaction cross sections $[50,51]$. This computational framework includes necessary input on nuclear structure properties, optical models, level densities, fission properties, etc. [51]. 
Nuclear level densities along with optical model transmission coefficients are considered as two most important ingredients of the statistical model. The knowledge on all relevant physical quantities and properties of target nuclei, could be used from available experimental data or theoretical models. However, in the case of nuclei for which experimental data are limited, theoretical models represent the only possible source of relevant nuclear data. Since many statistical model ingredients are not available from the experiment, phenomenological models are often used instead [8].

The nuclear properties needed for this study, in particular nuclear masses and level densities, are calculated using the nuclear energy density fuctional (EDF) theory. This framework represents the most complete description of ground-state properties, collective excitations and processes over the whole nuclide chart, from relatively light systems to superheavy nuclei, and from the valley of stability to the drip-lines [60-72]. Among the microscopic approaches to the nuclear many-body problem, no other method achieves comparable global accuracy at the same computational cost. It is the only approach that can describe the evolution of nuclear structure throughout the nuclide chart. In practical implementations the nuclear EDF framework is analogous to Kohn-Sham density functional theory (DFT) $[73,74]$. It is a widely used method for electronic structure calculations in condensed matter physics and quantum chemistry. Energy density functionals have so far been constructed mostly empirically, with their parameters usually adjusted to properties of symmetric and asymmetric nuclear matter, and bulk properties of a set of nuclei.

For the purpose of the present work, two main approaches based on the TALYS code are employed $[50,51]$. In the first case the experimental masses are used, and the level densities are based on a Fermi gas model. We denote this setting as TALYS-a. The second approach is more consistent, based on microscopic theoretical description, that employs the Skyrme-type functional for the description of nuclear masses and level densities (in the following, denoted as TALYS-b). In particular, the Skyrme functional with improved description of pairing, HFB-17, is used. More details on the interaction parameters, constrained within Hartree-Fock-Bogoliubov model are available in ref. [52]. The Skyrme EDF has been in the past successfully employed in description of a variety of nuclear properties and astrophysically relevant processes. As pointed out in ref. [75], when dealing with nuclear astrophysics applications, various nuclear inputs should be, when possible, determined from global, universal and microscopic models. Therefore, the implementation of the Skyrme functional represents a reasonable and consistent approach in modeling the $(n, \alpha)$ reaction cross sections. The Hauser-Feshbach model also requires description of the transmission coefficients for the $\alpha$ particle emission. In this work we employ well established $\alpha$ optical model potential from ref. [76], where the parameterisation used are given in detail in the TALYS-1.95 implementation [77]. Compound nucleus cross section also includes width fluctuation correction (WFC) which accounts for the correlations between the incident and outgoing waves [77]. These correlations enhance the elastic channel, and accordingly decrease other open channels. In this work the WFC factors are calculated using the Moldauer model [78]. With these settings, we use the TALYS-1.95 code [77] to calculate the $(n, \alpha)$ reaction cross sections, as well as the Maxwellian averaged cross sections (MACS) of particular relevance for the weak s-process nucleosynthesis and future experimental studies of $(n, \alpha)$ reactions at novel research facilities.

\section{Results and Discussion}

\section{1. $(n, \alpha)$ Reaction Cross Sections for s-Process Nuclei}

The theory framework outlined in Section 2 is employed in the study of $(n, \alpha)$ reaction cross sections for the set of nuclei involved in the weak s-process [25]. Figure 1 shows the $(n, \alpha)$ reaction cross sections for the set of twelve nuclei introduced in Section 1 . For the sake of completeness, we present not only astrophysically relevant low-energy range of the cross sections, emphasized by using logarithmic scale, but a complete result. At low energies, $E<100 \mathrm{keV}$, the cross sections show the $1 / v$ dependence that is a general feature in neutron induced reactions in this energy range [79]. At the intermediate energies, all 
of the cross sections are peaked within the energy range $E \approx 1-20 \mathrm{MeV}$. Their maximal values vary from $\approx 30 \mathrm{mb}$ for ${ }^{71} \mathrm{Ge}$ toward more than $300 \mathrm{mb}$ for ${ }^{41} \mathrm{Ca}$ and ${ }^{18} \mathrm{~F}$. The shape of the cross sections corresponds to the general expectation for the compound nucleus reaction, i.e., the $(n, \alpha)$ reaction cross section increases to a maximum and then decreases because higher energies start opening new emission channels [79]. The details of the cross sections sensitively depend on the properties of nuclei involved in reactions, in particular on their masses, excitation spectra and level densities. Clearly, the variations of the cross sections for presented nuclei indicate the necessity for systematic calculations that include all relevant nuclear properties.

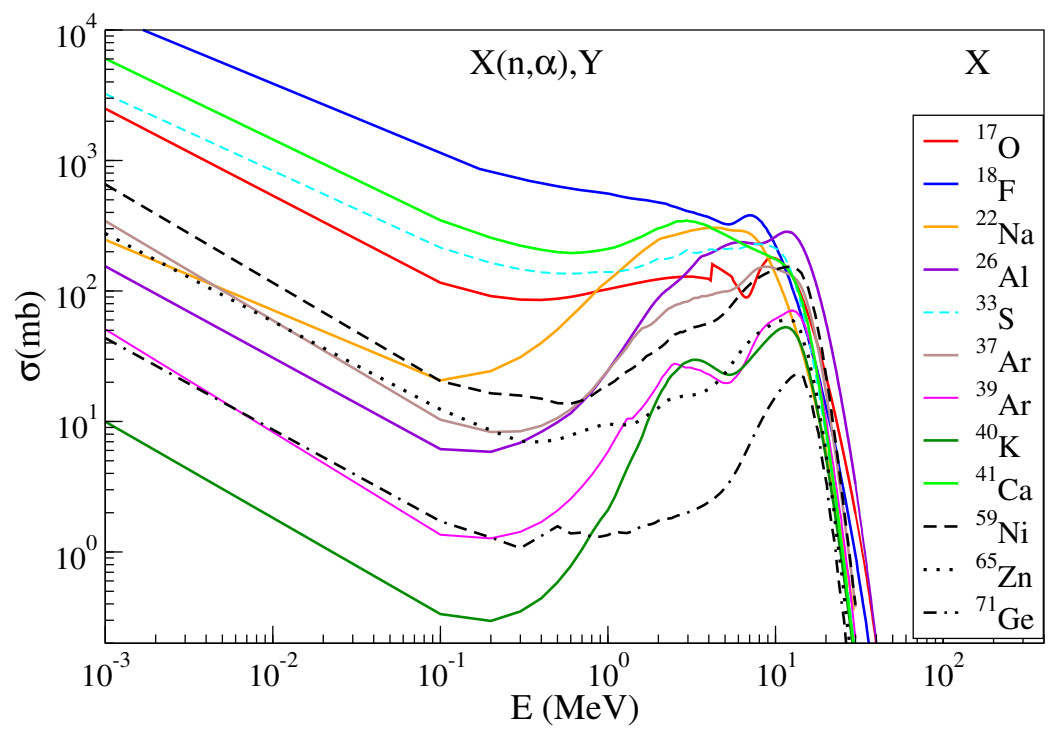

Figure 1. The $(n, \alpha)$ reaction cross section as a function of the incoming neutron energies for target nuclei as listed in the figure.

Model calculations are especially important for ${ }^{18} \mathrm{~F},{ }^{22} \mathrm{Na},{ }^{39} \mathrm{Ar},{ }^{40} \mathrm{~K},{ }^{65} \mathrm{Zn}$ and ${ }^{71} \mathrm{Ge}$, because no experimental data are existing on $(n, \alpha)$ reactions for these nuclei, or they are very limited. For other s-process nuclei studied in this work, some experimental data are available in the restricted low-energy range [26,35,37,38,80-82]. Figure 2 shows the $(n, \alpha)$ reaction cross sections for ${ }^{17} \mathrm{O},{ }^{26} \mathrm{Al},{ }^{33} \mathrm{~S},{ }^{37} \mathrm{Ar}$, and ${ }^{41} \mathrm{Ca}$ target nuclei, where the experimental data exist in the low-energy range [26,35,37,81,82]. Two different Hauser-Feshbach model calculations have been performed, (i) with experimental masses and level densities from the Fermi gas model (TALYS-a) and (ii) nuclear masses and level densities calculated with the Skyrme functional (TALYS-b), with the exception of ${ }^{17} \mathrm{O}$, where the experimental mass is used, due to the reasons discussed above. In this way one can also obtain the information on the sensitivity of the cross sections on these two essential ingredients in modeling reaction cross sections, i.e., up to an order of magnitude difference is obtained between the results of these two calculations. Nevertheless, the cross sections correspond reasonably well to the experimental data for ${ }^{26} \mathrm{Al}[35],{ }^{33} \mathrm{~S}[81,82],{ }^{37} \mathrm{Ar}$ [37], and ${ }^{41} \mathrm{Ca}$ [26]. For comparison, Figure 2 also shows the results from the evaluated data sets NON-SMOKER [8] TENDL2019 [83], ENDF-B-VIII [84], JEFF-3.3 [9] and BROND-3.1 [85]. These data sets include results of Hauser-Feshbach model calculations, however, in comparison to the present work, they are based on different selections of nuclear masses, excitation spectra, level densities, optical potential, WFC factors, and as a result the corresponding cross sections are subject to variations depending on the nuclear input. Figure 2 shows that in most of the cases, reasonable agreement is obtained between the present results and experimental data. Comparison with other evaluated data display qualitative agreement though some systematic differences are obtained, except at lower-end energies where the TENDL-2019 data show rapid decrease of the cross sections for several orders of magnitude (except for $\left.{ }^{37} \mathrm{Ar}\right)$. There is no reported explanation for the strong kink observed for the TENDL-2019 
cross sections. Although experimental data also exist for the ${ }^{59} \mathrm{Ni}$ cross section [38,44], these are not shown here because of a single resonance peak structure obtained at $203 \mathrm{eV}$ excitation energy, for which the Hauser-Feshbach model is not applicable. For ${ }^{40} \mathrm{~K}$, only a single data point is available, at very low energy, $0.025 \mathrm{eV}$. Thus, novel experimental studies are needed over a broader astrophysically relevant energy range that would also allow to constrain and improve model calculations.
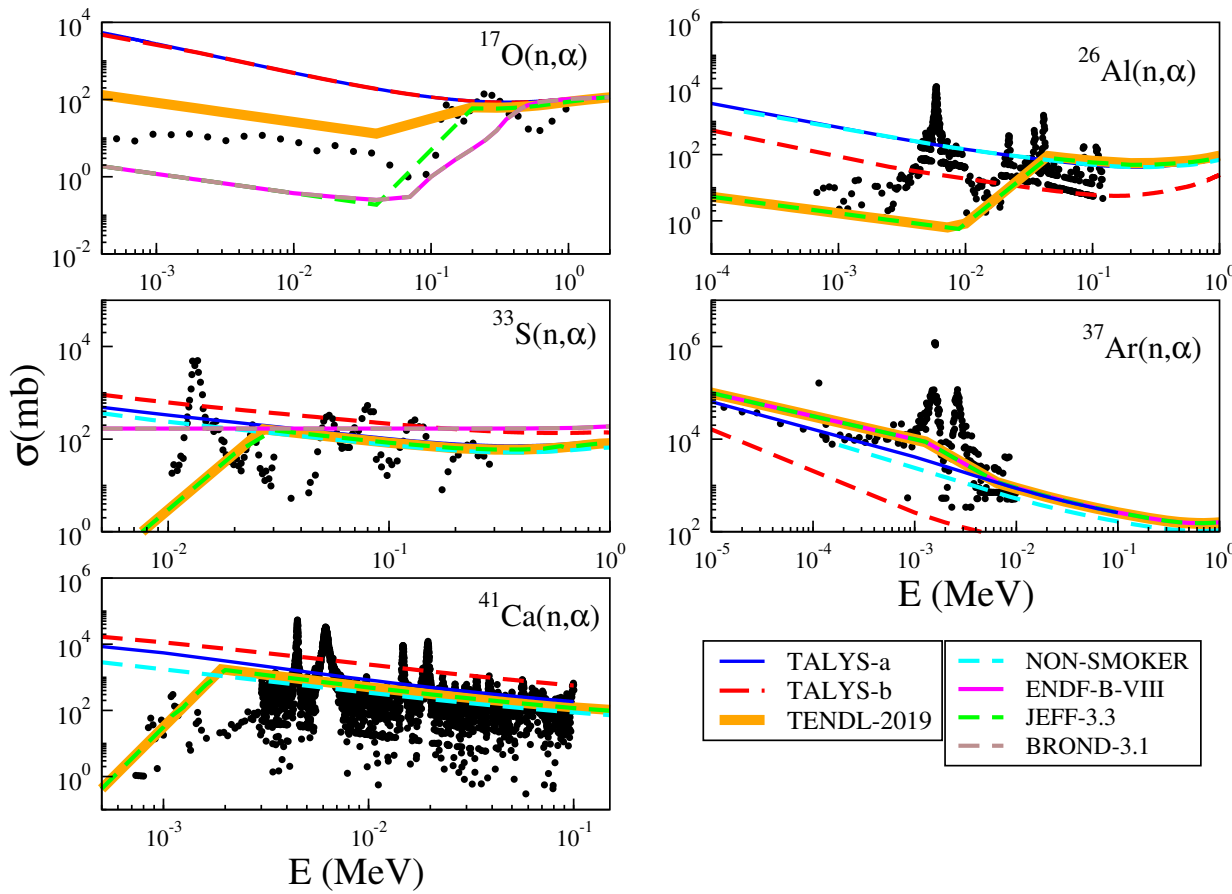

Figure 2. Comparison between the calculated and experimental $(n, \alpha)$ reaction cross sections for ${ }^{17} \mathrm{O},{ }^{26} \mathrm{Al},{ }^{33} \mathrm{~S},{ }^{37} \mathrm{Ar}$, and ${ }^{41} \mathrm{Ca}$. Model calculations include experimental nuclear masses and level densities from the Fermi gas model (TALYS-a) and nuclear masses and level densities from the Skyrme functional (TALYS-b). For comparison, results from the NON-SMOKER [8] TENDL-2019 [83], ENDF-B-VIII [84], JEFF-3.3 [9] and BROND-3.1 [85] data sets are also shown. The experimental data are taken from refs. $[26,35,37,81,82]$.

\subsection{Astrophysically Relevant Neutron Energy Window for $(n, \alpha)$ Reactions}

Next we explore the $(n, \alpha)$ reaction cross sections relevant for the s-process nucleosynthesis in stellar environment. For this purpose the cross sections are averaged over the Maxwell-Boltzmann distribution, that describes the distribution of the neutron energies with respect to the corresponding temperature [30],

$$
\langle\sigma\rangle(k T)=\frac{2}{\sqrt{\pi}}(k T)^{-2} \int_{0}^{\infty} \sigma(E) E e^{-\frac{E}{k T}} d E,
$$

where $k$ and $T$ denote the Boltzmann constant and temperature, respectively. Figure 3 shows the Mawellian averaged $(n, \alpha)$ reaction cross sections (MACS) [30] obtained for the same set of nuclei discussed in Section 3.1. In the astrophysically relevant low-temperature range, one can observe a systematic decrease of the MACS values, that is governed by the $1 / v$ dependence of the cross sections shown in Figure 1. Following the Gaussian-like shape of the cross sections at intermediate energies, $E \approx 1-30 \mathrm{MeV}$ (see Figure 1 ), the corresponding MACS values start to increase in the temperature range $k T \approx 100 \mathrm{keV}-1 \mathrm{MeV}$. Model calculations also identify a hierarchy of the MACS values, showing the largest cross sections for the four target nuclei, ${ }^{18} \mathrm{~F},{ }^{41} \mathrm{Ca}$ and ${ }^{33} \mathrm{~S}$, and ${ }^{17} \mathrm{O}$ that reflect large cross sections in the low energy region shown in Figure 1. The MACS values for other nuclei appear in most of the cases an order of magnitude smaller. The resulting behaviour of the MACS values 
could have implications in network calculations of the weak s-process nucleosynthesis, identifying relevant contributions from $(n, \alpha)$ reactions for nuclei where the corresponding cross sections have larger values. However, these cross sections need to be considered in competition with neutron induced reactions with other possible exit channels.

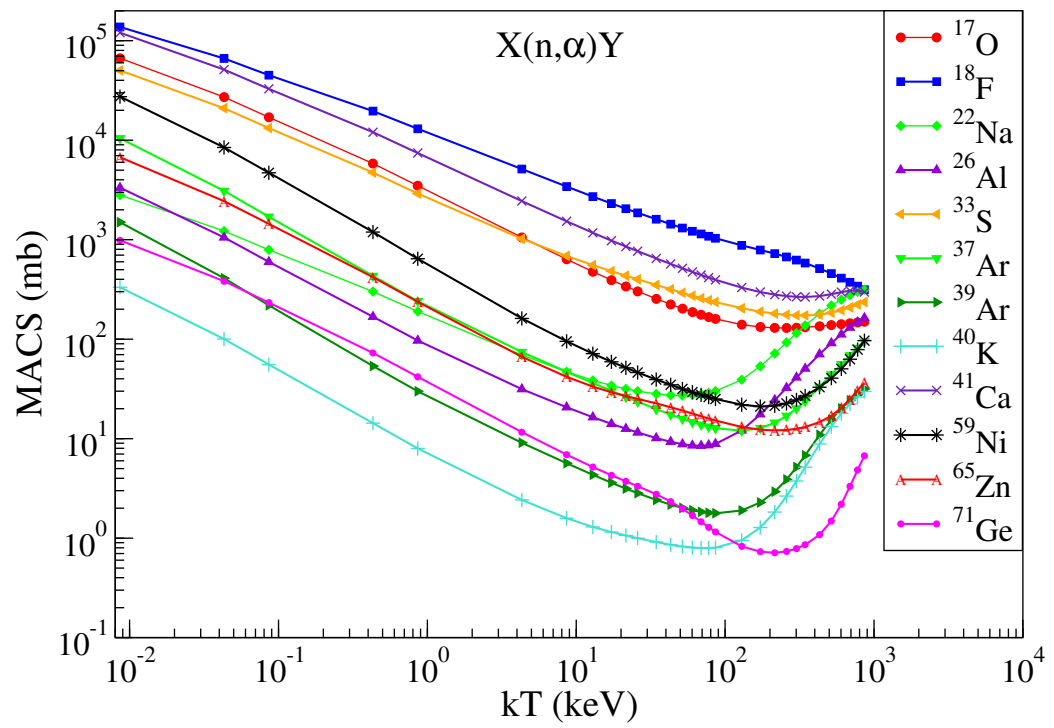

Figure 3. The $(n, \alpha)$ reaction cross sections averaged over the Maxwell-Boltzmann distribution for the set of nuclei shown as a function of temperature.

In order to assess the information on the systematic uncertainties in modeling $(n, \alpha)$ reactions, it is necessary to compare the results from various theoretical approaches based on different assumptions. In ref. [86] the variations of theoretical results have been explored for ${ }^{26} \mathrm{Al}(n, \alpha){ }^{23} \mathrm{Na}$ reaction cross sections when using three different approaches, TALYS [50], EMPIRE [87], and NON-SMOKER [8]. Figure 4 shows the MACS results of the present work for ${ }^{22} \mathrm{Na},{ }^{26} \mathrm{Al}$, and ${ }^{33} \mathrm{~S},{ }^{37} \mathrm{Ar}$, and ${ }^{39} \mathrm{Ar}$ target nuclei in comparison with those from several other model calculations based on different assumptions on the nuclear properties and approaches in modeling the reaction mechanism. These include NON-SMOKER reaction cross sections data base [8], Evaluated Nuclear Data File (ENDF-B-VII.1) [7], Joint Evaluated Fission and Fusion (JEFF-3.1) Nuclear Data Library [30], JENDL-4.0 Library for Nuclear Science and Engineering [10], and ROSFOND-2008 nuclear data [11]. The MACS results from this work are in qualitative agreement with the Hauser-Feshbach calculation NON-SMOKER for ${ }^{22} \mathrm{Na}$ (except for $k T>100 \mathrm{keV}$ ), ${ }^{26} \mathrm{Al}$, and ${ }^{39} \mathrm{Ar}$, while for ${ }^{33} \mathrm{~S}$ and ${ }^{37}$ Ar even more than an order of magnitude difference is obtained. Agreement with the ENDF, JEFF and JENDL evaluation data depends on the range of $k T$ values of interest. For example, the MACS values for ${ }^{33} \mathrm{~S}$ in this work, as well as those from NON-SMOKER, are considerably larger than ENDF, JEFF, and JENDL evaluation data for $k T<10 \mathrm{keV}$. In the case of ${ }^{26} \mathrm{Al},{ }^{33} \mathrm{~S}$, and ${ }^{39} \mathrm{Ar}$, for $k T<100 \mathrm{keV}$, large discrepancies are obtained between the present MACS values (as well as those from other models) and the ROSFOND evaluation data, which also show different trend with increasing temperature. Clearly, different foundations in various model calculations result in considerable model dependence of the MACS values.

Figure 5 shows the MACS values for ${ }^{40} \mathrm{~K},{ }^{41} \mathrm{Ca},{ }^{59} \mathrm{Ni},{ }^{65} \mathrm{Zn}$, and ${ }^{71} \mathrm{Ge}$ target nuclei. In addition to the comparison with other evaluations as shown before, for ${ }^{65} \mathrm{Zn}$ the results from the Chinese Evaluated Nuclear Data Library (CENDL-3.1) are also displayed. For all nuclei except ${ }^{40} \mathrm{~K}$, good qualitative agreement of TALYS-b MACS values with other evaluations is obtained, except for the ROSFOND-2008 which result in considerably smaller values except for ${ }^{40} \mathrm{~K}$. The differences obtained from comparison of the cross sections from various theoretical frameworks demonstrate the necessity for calculations of the MACS values 
using different approaches and parameterizations, and analyses of their impact in the network calculations of the s-process nucleosynthesis in realistic stellar conditions.

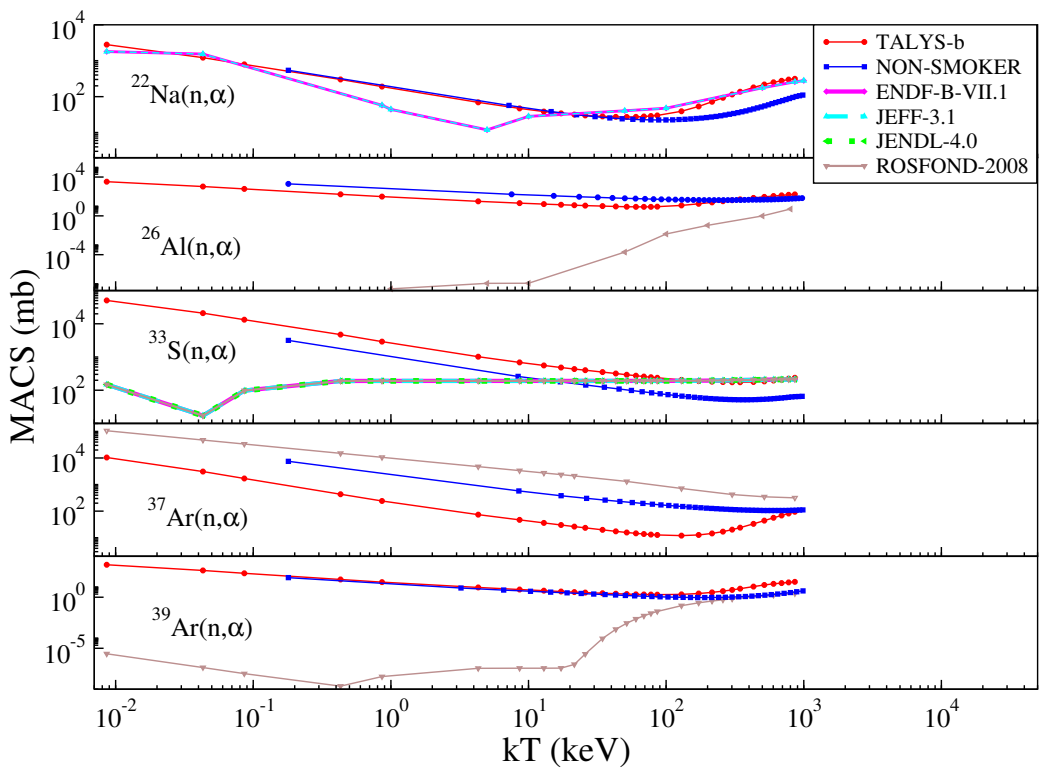

Figure 4. The Maxwellian averaged cross sections (MACS) for $(n, \alpha)$ reaction for ${ }^{22} \mathrm{Na},{ }^{26} \mathrm{Al}$, and ${ }^{33} \mathrm{~S}$, ${ }^{37} \mathrm{Ar}$, and ${ }^{39} \mathrm{Ar}$ as functions of temperature. The present results (TALYS-b) are compared to those from NON-SMOKER [8], ENDF-B-VII.1 [7], JEFF-3.1 [30], JENDL-4.0 [10], and ROSFOND-2008 [11].

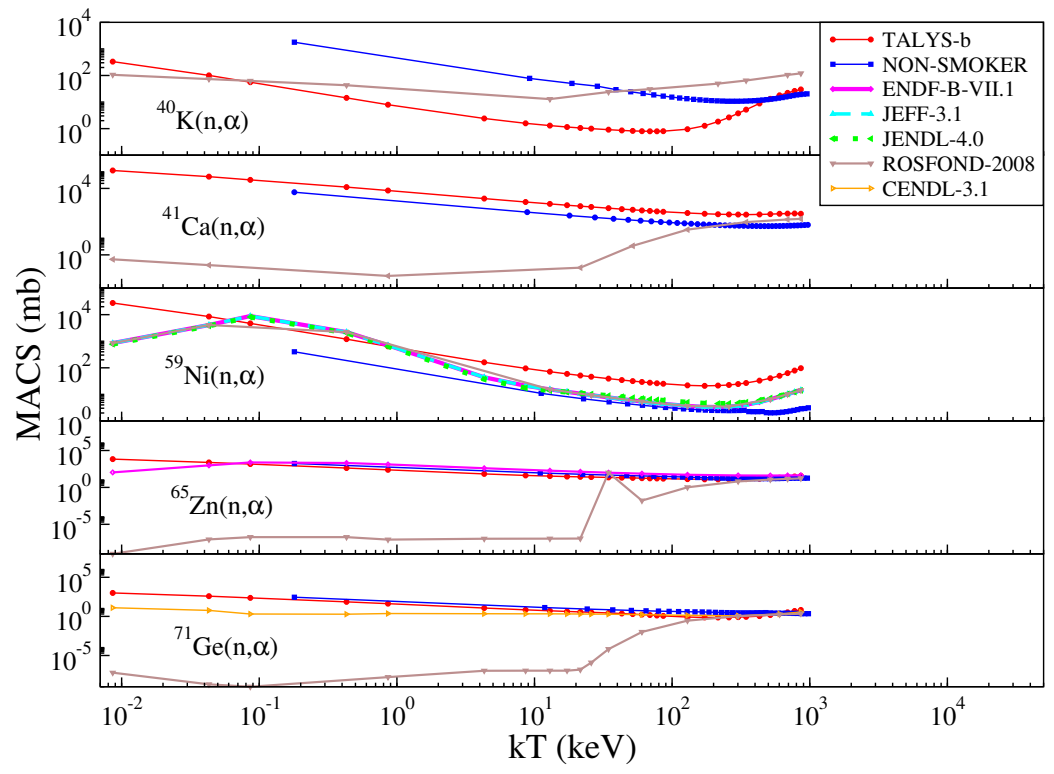

Figure 5. The same as in Figure 4, but for ${ }^{40} \mathrm{~K},{ }^{41} \mathrm{Ca},{ }^{59} \mathrm{Ni},{ }^{65} \mathrm{Zn}$, and ${ }^{71} \mathrm{Ge}$ target nuclei. In addition to the results from other data sets, comparison with the MACS values from the CENDL-3.1 [12] nuclear data library is also shown.

\subsection{Astrophysically Relevant Neutron Energy Range for $(n, \alpha)$ Reactions}

As shown in Section 3.2, the $(n, \alpha)$ reaction cross sections are subject to a considerable model dependence. In order to have more stringent constraints on the astrophysically relevant neutron induced reactions, novel experimental studies are needed. As discussed in Section 3.1 available experimental data on s-process nuclei studied in this work are rather limited. One of the objectives of this study is to determine what are the astrophysically relevant energy ranges that could contribute in modeling the nucleosynthesis. These relevant energy ranges, known as Gamow window, are determined as the overlap region 
between the Maxwell Boltzmann distribution of the interacting particles and the low-energy tail of the reaction cross section [55]. The exact position of the Gamow window depends on typical temperature regimes characteristic for a specific core and burning stages during stellar evolution. While in the case of reactions that involve the charge particles, the Gamow peak is determined by the interplay of the Maxwell-Boltzmann distribution and tunneling of the incoming particle throught the Coulomb barrier of target nucleus [58], in the case of $(n, \alpha)$ reactions there is no Coulomb barrier for the incoming particle and the relevant energy range is determined by the overlap of the Maxwell-Boltzmann distribution and the reaction cross section. This is illustrated in Figure 6 which shows the contributions from the Maxwell-Boltzmann distribution $f_{D}(E)$ (in arbitrary units for the presentation purposes) and $(n, \alpha)$ reaction cross sections for ${ }^{41} \mathrm{Ca},{ }^{59} \mathrm{Ni},{ }^{65} \mathrm{Zn}$, and ${ }^{71} \mathrm{Ge}$ as a function of neutron energy. The distributions are displayed for the range of temperatures $k T=30-210 \mathrm{keV}$. For these temperatures the Maxwell-Boltzmann distributions limit the neutron energy range at low energies. For comparison, the $(n, \alpha)$ cross sections are also shown. Thus, only the low-energy part of the cross sections are relevant for astrophysical applications within the temperature range as given above.

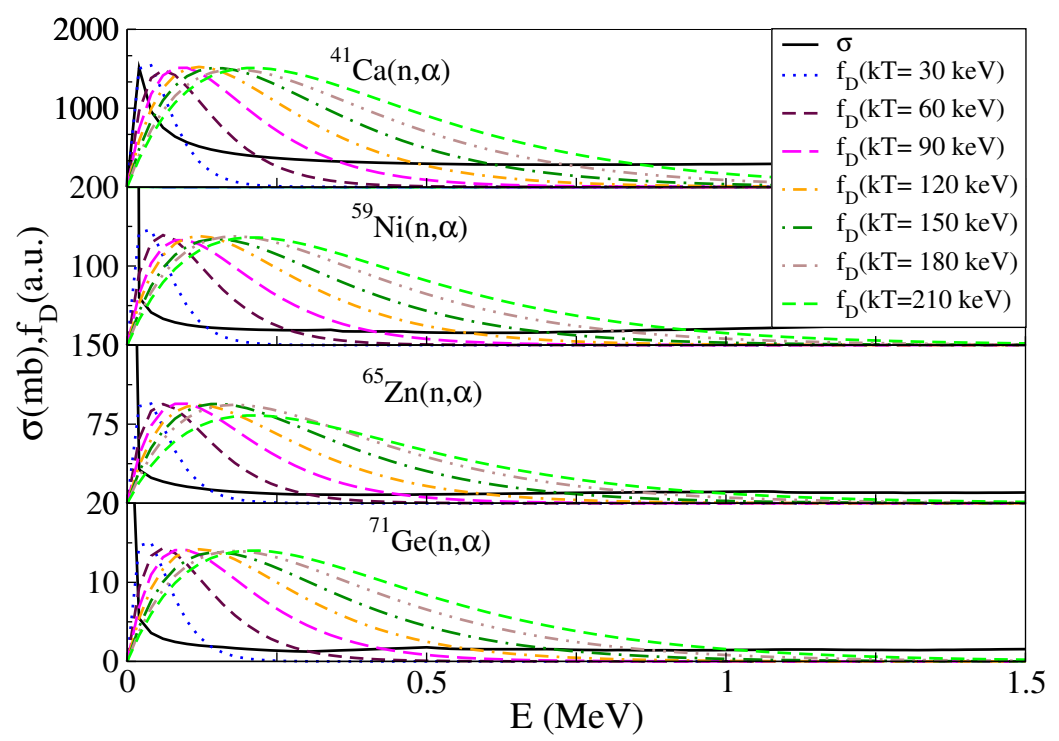

Figure 6. The $(n, \alpha)$ reaction cross sections as a function of neutron energy $\sigma(E)$, and MaxwellBoltzmann distribution $f_{D}(E)$ (in arbitrary units) for the range of temperatures $k T=30-210 \mathrm{keV}$, shown for ${ }^{41} \mathrm{Ca},{ }^{59} \mathrm{Ni},{ }^{65} \mathrm{Zn}$, and ${ }^{71} \mathrm{Ge}$.

In the following we determine the relevant energy windows for $(n, \alpha)$ reactions for the complete previously studied set of nuclei. For this purpose, we analyze the subintegral function used in calculating the MACS values (1),

$$
f_{I}(E)=\sigma(E) E e^{-E /(k T)},
$$

that provides the cross section weighted by the corresponding Maxwell-Boltzmann distribution at a given energy. The reaction energy windows described by the function $f_{I}(E)$, together with the corresponding $(n, \alpha)$ reaction cross sections are shown in Figure 7 for ${ }^{17} \mathrm{O},{ }^{18} \mathrm{~F},{ }^{22} \mathrm{Na}$, and ${ }^{26} \mathrm{Al}$. As expected, the reaction energy windows become wider with increasing temperature, but their exact location sensitively depends on the specific target nucleus under consideration. For all nuclei, the maximal reaction peaks for $k T=30 \mathrm{keV}$ are located below energy of $0.1 \mathrm{MeV}$. To assess the accurate information on astrophysically relevant energy windows, calculations need to be performed for each nucleus of interest. Therefore, we show the results for relevant $(n, \alpha)$ reaction windows also for ${ }^{33} \mathrm{~S}$, ${ }^{37} \mathrm{Ar},{ }^{39} \mathrm{Ar}$, and ${ }^{40} \mathrm{~K}$ in Figure 8 and for ${ }^{41} \mathrm{Ca},{ }^{59} \mathrm{Ni},{ }^{65} \mathrm{Zn},{ }^{71} \mathrm{Ge}$ in Figure 9 . The results give quantitative predictions which energy windows are important for different nuclei within 
given temperature range, and in this way provide a guidance for the future experimental studies of $(n, \alpha)$ reactions, and their implementation in the s-process network calculations. As already discussed in Section 3.2, considerable model dependence of $(n, \alpha)$ reaction cross sections necessitate more experimental data that could provide additional constraints on these reactions that contribute to the s-process nucleosynthesis.

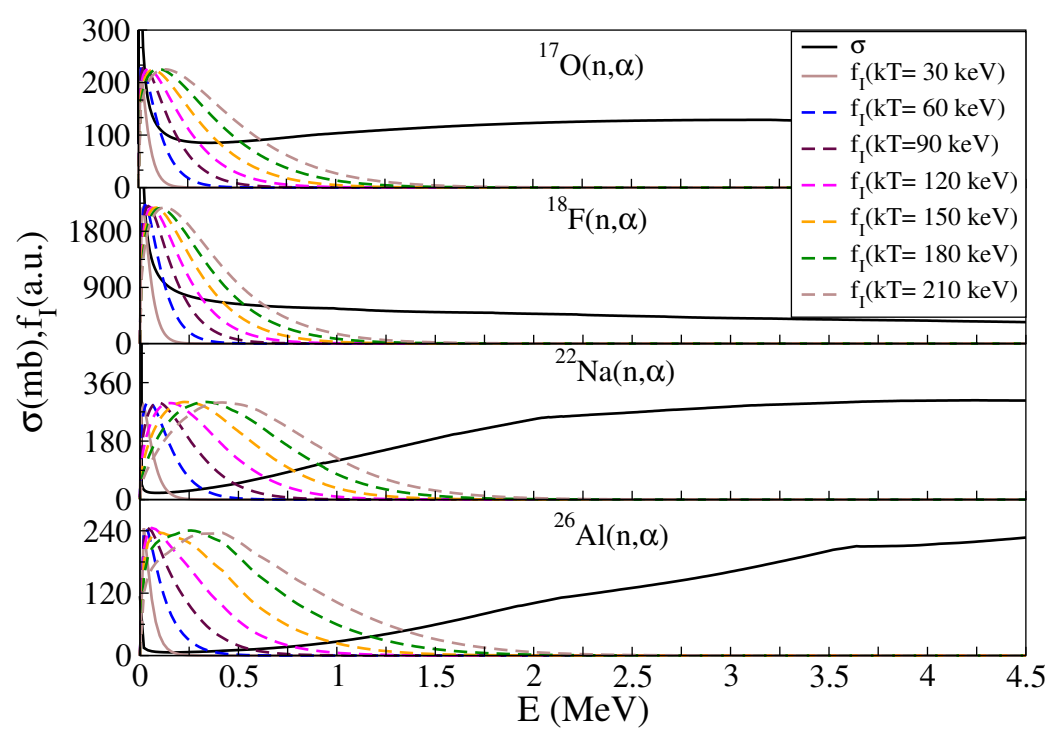

Figure 7. Relevant $(n, \alpha)$ reaction energy windows for the range of temperatures $k T=30-210 \mathrm{keV}$ described with the function $f_{I}(E)$ (in arbitrary units, see text) and the respective reaction cross sections for ${ }^{17} \mathrm{O},{ }^{18} \mathrm{~F},{ }^{22} \mathrm{Na}$, and ${ }^{26} \mathrm{Al}$ target nuclei.

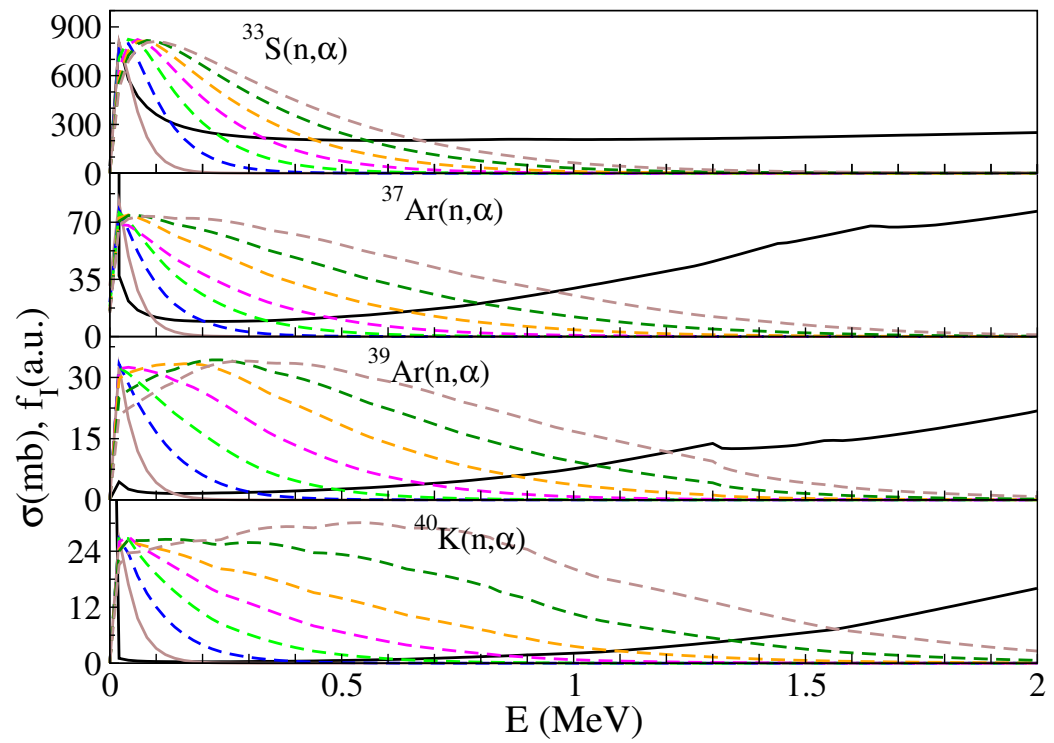

Figure 8. The same as Figure 7, but for ${ }^{33} \mathrm{~S},{ }^{37} \mathrm{Ar},{ }^{39} \mathrm{Ar}$, and ${ }^{40} \mathrm{~K}$ target nuclei. 


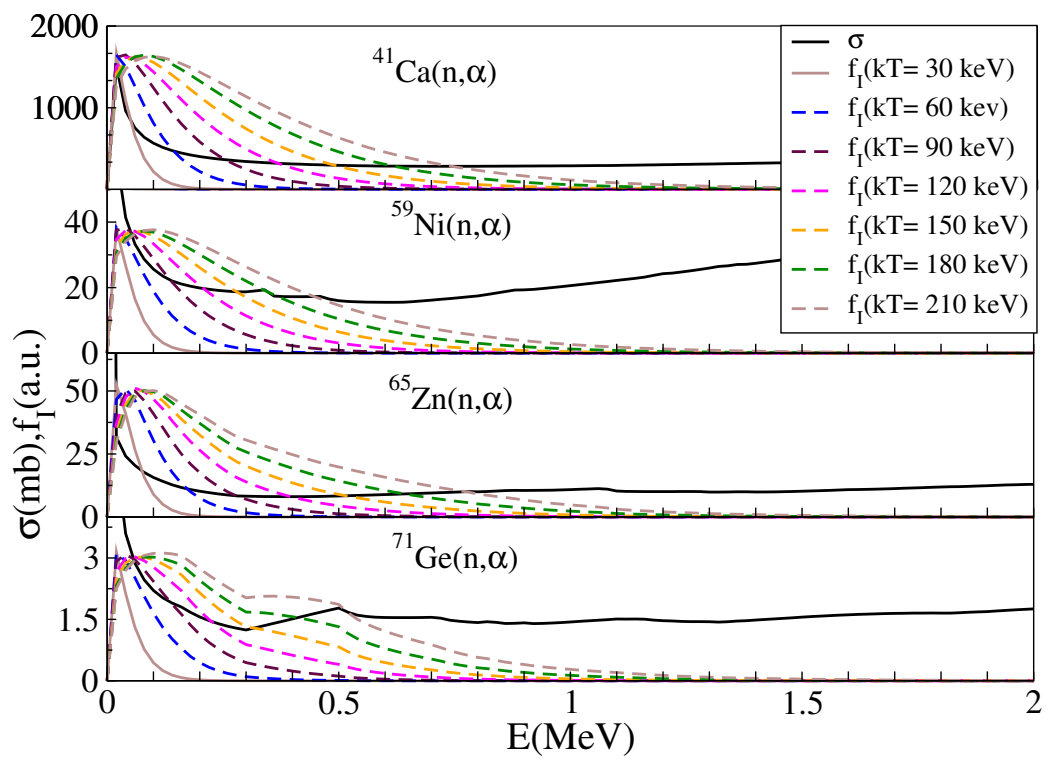

Figure 9. The same as Figure 7 , but for ${ }^{41} \mathrm{Ca},{ }^{59} \mathrm{Ni},{ }^{65} \mathrm{Zn}$, and ${ }^{71} \mathrm{Ge}$ target nuclei.

\section{Conclusions}

In this work we have investigated $(n, \alpha)$ reaction cross sections for the set of nuclei contributing to the s-process nucleosynthesis. Model calculations have been performed in the theory framework based on Hauser-Feshbach statistical model through its implementation in the nuclear reaction program TALYS-1.95 [50]. When possible, model calculations are kept consistent by using nuclear properties necessary for the reaction calculated in the framework of Skyrme energy density functional. The $(n, \alpha)$ reactions have been investigated in view of their relevance for the weak s-process nucleosynthesis, thus their average over the Maxwell-Boltzmann distribution for the range of stellar temperatures has been calculated.

An important contribution of this work is a quantitative assessment of the energy window in which $(n, \alpha)$ reactions occur in stars, that is by its role equivalent to the well known Gamow window, with a difference that in the case of $(n, \alpha)$ reactions no tunneling through the Coulomb barrier is needed in the entrance channel. Model calculations in this work determined relevant energy windows for $(n, \alpha)$ reactions for the range of astrophysically represented temperatures, indicating a strong dependence of the exact location of the energy window on specific target nucleus under consideration. Quantitative predictions of astrophysically relevant energy windows for $(n, \alpha)$ reactions, that contribute in the s-process nucleosynthesis, provide a guidance for the future experimental studies of these reactions. In particular, measurements in the energy windows as identified in Figures 7-9 are necessary, especially for nuclei for which none or very limited data exist, ${ }^{18} \mathrm{~F},{ }^{22} \mathrm{Na},{ }^{39} \mathrm{Ar},{ }^{40} \mathrm{~K},{ }^{59} \mathrm{Ni},{ }^{65} \mathrm{Zn}$, and ${ }^{71} \mathrm{Ge}$. As shown in this study, $(n, \alpha)$ reactions, similar as all other neutron induced reactions, are subject to a considerable systematic model dependence. Therefore, novel experimental data are necessary in the predicted relevant energy windows, that could provide additional constraints on these reactions and reduce currently existing theoretical uncertainties. In the forthcoming studies the $(n, \alpha)$ reaction cross sections obtained in this work could also be implemented in the s-process network calculations to explore the variations of isotopes in the element abundances.

Author Contributions: Conceptualization, N.P. and S.K.; methodology, S.K. and N.P.; software, S.K.; validation, S.K., M.Y. and N.P.; formal analysis, S.K.; investigation, S.K. and N.P.; writing-original draft preparation, S.K. and N.P.; writing-review and editing, M.Y. and N.P.; visualization, S.K.; supervision, N.P. and M.Y.; project administration, S.K.; funding acquisition, S.K. and N.P. All authors have read and agreed to the published version of the manuscript. 
Funding: This research was funded by QuantiXLie Centre of Excellence, a project co financed by the Croatian Government and European Union through the European Regional Development Fund, the Competitiveness and Cohesion Operational Programme (KK.01.1.1.01). S.K. acknowledges support from the Scientific and Technological Research Council of Turkey (TUBITAK) through the International Doctoral Research Fellowship Programme 2214A, 2020/1, Grant No. 1059B142000254.

Acknowledgments: We thank M. Milin and P. Žugec for useful discussions on neutron induced reactions.

Conflicts of Interest: The authors declare no conflict of interest. The funders had no role in the design of the study; in the collection, analyses, or interpretation of data; in the writing of the manuscript, or in the decision to publish the results.

\section{References}

1. Burbidge, E.M.; Burbidge, G.R.; Fowler, W.A.; Hoyle, F. Synthesis of the Elements in Stars. Rev. Mod. Phys. 1957, 29, 547-650. [CrossRef]

2. Cameron, A.G.W. Stellar Evolution, Nuclear Astrophysics, and Nucleogenesis; Chalk River Report; Atomic Energy of Canada Ltd.: Chalk River, ON, Canada, 1957; Volume 41.

3. Janka, H.T.; Langanke, K.; Marek, A.; Martínez-Pinedo, G.; Müller, B. Theory of core-collapse supernovae. Phys. Rep. 2007, $442,38-74$. [CrossRef]

4. José, J.; Iliadis, C. Nuclear astrophysics: The unfinished quest for the origin of the elements. Rep. Prog. Phys. 2011, 74, 096901. [CrossRef]

5. Bracco, A.; Körner, G.E.; Krusche, B.; Nappi, E.; Maj, A.; Murphy, A.; Nystrand, J.; Widmann, E.; Dobeš, J.; Lewitowicz, M. (Eds.) NuPECC Long Range Plan 2017 Perspectives in Nuclear Physics; Nuclear Physics European Collaboration Committee: Caen, France, 2017; p. 236. Available online: https://www.esf.org/fileadmin/user_upload/esf/Nupecc-LRP2017.pdf (accessed on 28 November 2021).

6. Otuka, N.; Dupont, E.; Semkova, V.; Pritychenko, B.; Blokhin, A.; Aikawa, M.; Babykina, S.; Bossant, M.; Chen, G.; Dunaeva, S.; et al. Towards a More Complete and Accurate Experimental Nuclear Reaction Data Library (EXFOR): International Collaboration Between Nuclear Reaction Data Centres (NRDC). Nucl. Data Sheets 2014, 120, 272-276. [CrossRef]

7. Chadwick, M.B.; Herman, M.; Obložinský, P.; Dunn, M.E.; Danon, Y.; Kahler, A.C.; Smith, D.L.; Pritychenko, B.; Arbanas, G.; Arcilla, R.; et al. ENDF/B-VII.1 Nuclear Data for Science and Technology: Cross Sections, Covariances, Fission Product Yields and Decay Data. Nucl. Data Sheets 2011, 112, 2887-2996. [CrossRef]

8. Rauscher, T.; Thielemann, F.K. Astrophysical Reaction Rates From Statistical Model Calculations. At. Data Nucl. Data Tables 2000, 75, 1-351. [CrossRef]

9. Plompen, A.J.M.; Cabellos, O.; De Saint Jean, C.; Fleming, M.; Algora, A.; Angelone, M.; Archier, P.; Bauge, E.; Bersillon, O.; Blokhin, A.; et al. The joint evaluated fission and fusion nuclear data library, JEFF-3.3. Eur. Phys. J. A 2020, 56, 181. [CrossRef]

10. Shibata, K.; Iwamoto, O.; Nakagawa, T.; Iwamoto, N.; Ichihara, A.; Kunieda, S.; Chiba, S.; Furutaka, K.; Otuka, N.; Ohsawa, T.; et al. JENDL-4.0: A New Library for Nuclear Science and Engineering. J. Nucl. Sci. Technol. 2011, 48, 1-30. [CrossRef]

11. Andrianova, O.N.; Golovko, Y.Y.; Manturov, G.N. Verification of the ROSFOND/ABBN nuclear data based on the OECD/NEA benchmark on criticality safety of mox-fueled systems. Nucl. Energy Technol. 2019, 5, 91-96. [CrossRef]

12. Ge, Z.; Zhao, Z.; Xia, H.; Zhuang, Y.; Liu, T.; Zhang, J.; Wu, H. The Updated Vversion of Chinese Evaluated Nuclear Data Library (CENDL-3.1). J. Korean Phys. Soc. 2011, 59, 1052-1056. [CrossRef]

13. Bao, Z.; Käppeler, F. Neutron capture cross sections for s-process studies. At. Data Nucl. Data Tables 1987, 36, 411-451. [CrossRef]

14. Käppeler, F.; Gallino, R.; Bisterzo, S.; Aoki, W. The s process: Nuclear physics, stellar models, and observations. Rev. Mod. Phys. 2011, 83, 157-193. [CrossRef]

15. Banerjee, P.; Heger, A.; Qian, Y.Z. New s-process Mechanism in Rapidly Rotating Massive Population II Stars. Astrophys. J. 2019, 887, 187. [CrossRef]

16. Mathews, G.J.; Cowan, J.J. New insights into the astrophysical r-process. Nature 1990, 345, 491-494. [CrossRef]

17. Cowan, J.J.; Thielemann, F.K.; Truran, J.W. The R-process and nucleochronology. Phys. Rep. 1991, 208, 267-394. [CrossRef]

18. Wanajo, S.; Tamamura, M.; Itoh, N.; Nomoto, K.; Ishimaru, Y.; Beers, T.C.; Nozawa, S. Ther-Process in Supernova Explosions from the Collapse of O-Ne-Mg Cores. Astrophys. J. 2003, 593, 968-979. [CrossRef]

19. Thielemann, F.K.; Arcones, A.; Käppeli, R.; Liebendörfer, M.; Rauscher, T.; Winteler, C.; Fröhlich, C.; Dillmann, I.; Fischer, T.; Martinez-Pinedo, G.; et al. What are the astrophysical sites for the r-process and the production of heavy elements? Prog. Part. Nucl. Phys. 2011, 66, 346-353. [CrossRef]

20. Freiburghaus, C.; Rosswog, S.; Thielemann, F.K. r-Process in Neutron Star Mergers. Astrophys. J. 1999, 525, L121-L124. [CrossRef]

21. Goriely, S.; Bauswein, A.; Janka, H.T. r-process nucleosynthesis in dynamically ejected matter of neutron star mergers. Astrophys. J. Lett. 2011, 738, L32. [CrossRef]

22. Pian, E.; D'Avanzo, P.; Benetti, S.; Branchesi, M.; Brocato, E.; Campana, S.; Cappellaro, E.; Covino, S.; D’Elia, V.; Fynbo, J.P.U.; et al. Spectroscopic identification of r-process nucleosynthesis in a double neutron-star merger. Nature 2017, 551, 67-70. [CrossRef] 
23. Kajino, T.; Aoki, W.; Balantekin, A.; Diehl, R.; Famiano, M.; Mathews, G. Current status of r-process nucleosynthesis. Prog. Part. Nucl. Phys. 2019, 107, 109-166. [CrossRef]

24. Litvinova, E.; Loens, H.; Langanke, K.; MartÃnez-Pinedo, G.; Rauscher, T.; Ring, P.; Thielemann, F.K.; Tselyaev, V. Low-lying dipole response in the relativistic quasiparticle time blocking approximation and its influence on neutron capture cross sections. Nucl. Phys. A 2009, 823, 26-37. [CrossRef]

25. Pignatari, M.; Gallino, R.; Heil, M.; Wiescher, M.; Käppeler, F.; Herwig, F.; Bisterzo, S. The weak s-process in massive stars and its dependence on the neutron capture cross sections. Astrophys. J. 2010, 710, 1557-1577. [CrossRef]

26. Vermote, S.; Wagemans, C.; De Smet, L.; Lampoudis, C.; Van Gils, J. Experimental determination of the ${ }^{41} \mathrm{Ca}(n, \alpha)^{38} \mathrm{Ar}$ reaction cross section up to $80 \mathrm{keV}$, and calculation of the Maxwellian averaged cross section at stellar temperatures. Phys. Rev. C 2012, 85, 015803. [CrossRef]

27. Woosley, S.; Fowler, W.A.; Holmes, J.; Zimmerman, B. Semiempirical thermonuclear reaction-rate data for intermediate-mass nuclei. At. Data Nucl. Data Tables 1978, 22, 371-441. [CrossRef]

28. Schatz, H.; Kaeppeler, F.; Koehler, P.E.; Wiescher, M.; Trautvetter, H.P. 17O(n, alpha )14C: Closure of a Primordial CNO Bi-Cycle? Astrophys. J. 1993, 413, 750. [CrossRef]

29. Bertulani, C.; Kajino, T. Frontiers in nuclear astrophysics. Prog. Part. Nucl. Phys. 2016, 89, 56-100. [CrossRef]

30. Pritychenko, B.; Mughaghab, S.; Sonzogni, A. Calculations of Maxwellian-averaged cross sections and astrophysical reaction rates using the ENDF/B-VII.0, JEFF-3.1, JENDL-3.3, and ENDF/B-VI.8 evaluated nuclear reaction data libraries. At. Data Nucl. Data Tables 2010, 96, 645-748. [CrossRef]

31. Dan, M.; Singh, G.; Chatterjee, R.; Shubhchintak. Neutron capture rates of ${ }^{18}$ C. Phys. Rev. C 2019, 99, 035801. [CrossRef]

32. Konobeyev, A.; Lunev, V.; Shubin, Y. Semi-empirical systematics for $(n, \alpha)$ reaction cross sections at the energy of $14.5 \mathrm{MeV}$. Nucl. Instrum. Methods Phys. Res. Sect. B Beam Interact. Mater. Atoms 1996, 108, 233-242. [CrossRef]

33. Forrest, R.A. Systematics of Neutron-Induced Threshold Reactions with Charged Products at about $145 \mathrm{MeV}$; Technical Report AERE-R-12419; UKAEA Atomic Energy Research Establishment: Abingdon, UK, 1986.

34. Fessler, A.; Wattecamps, E.; Smith, D.L.; Qaim, S.M. Excitation functions of $(n, 2 n),(n, p),(n, n p+p n+d)$, and $(n, \alpha)$ reactions on isotopes of chromium. Phys. Rev. C 1998, 58, 996-1004. [CrossRef]

35. Smet, L.D.; Wagemans, C.; Goeminne, G.; Heyse, J.; Gils, J.V. Experimental determination of the ${ }^{36} \mathrm{Cl}(n, p)^{36} \mathrm{~S}$ and ${ }^{36} \mathrm{Cl}(n, \alpha)^{33} \mathrm{P}$ reaction cross sections and the consequences on the origin of ${ }^{36} \mathrm{~S}$. Phys. Rev. C 2007, 75, 034617. [CrossRef]

36. Gledenov, Y.M.; Koehler, P.E.; Andrzejewski, J.; Guber, K.H.; Rauscher, T. ${ }^{147} \mathrm{Sm}(n, \alpha)$ cross section measurements from 3 eV to $500 \mathrm{keV}$ : Implications for explosive nucleosynthesis reaction rates. Phys. Rev. C 2000, 62, 042801. [CrossRef]

37. Goeminne, G.; Wagemans, C.; Wagemans, J.; Serot, O.; Loiselet, M.; Gaelens, M. Investigation of the ${ }^{37} \mathrm{Ar}(n, p)^{37} \mathrm{Cl}$ and ${ }^{37} \operatorname{Ar}(n, \alpha)^{34} \mathrm{~S}$ reactions in the neutron energy range from $10 \mathrm{meV}$ to $100 \mathrm{keV}$. Nucl. Phys. A 2000, 678, 11-23. [CrossRef]

38. Weiß, C.; Guerrero, C.; Griesmayer, E.; Andrzejewski, J.; Badurek, G.; Chiaveri, E.; Dressler, R.; Ganesan, S.; Jericha, E.; Käppeler, F.; et al. The $(n, \alpha)$ Reaction in the s-process Branching Point ${ }^{59} \mathrm{Ni}$. Nucl. Data Sheets 2014, 120, 208-210. [CrossRef]

39. Fotiades, N.; Devlin, M.; Haight, R.C.; Nelson, R.O.; Kunieda, S.; Kawano, T. $\alpha$ and $2 p 2 n$ emission in fast neutron-induced reactions on ${ }^{60} \mathrm{Ni}$. Phys. Rev. C 2015, 91, 064614. [CrossRef]

40. Barbagallo, M.; Musumarra, A.; Cosentino, L.; Maugeri, E.; Heinitz, S.; Mengoni, A.; Dressler, R.; Schumann, D.; Käppeler, F.; Colonna, N.; et al. ${ }^{7} \mathrm{Be}(n, \alpha){ }^{4} \mathrm{He}$ Reaction and the Cosmological Lithium Problem: Measurement of the Cross Section in a Wide Energy Range at $n_{T}$ OF at CERN. Phys. Rev. Lett. 2016, 117, 152701. [CrossRef] [PubMed]

41. Gledenov, Y.M.; Sedysheva, M.V.; Khuukhenkhuu, G.; Bai, H.; Jiang, H.; Lu, Y.; Cui, Z.; Chen, J.; Zhang, G. Measurement of the cross sections of the ${ }^{25} \mathrm{Mg}(n, \alpha)^{22} \mathrm{Ne}$ reaction in the 4-6 MeV region. Phys. Rev. C 2018, 98, 034605. [CrossRef]

42. Praena, J.; Sabaté-Gilarte, M.; Porras, I.; Quesada, J.M.; Altstadt, S.; Andrzejewski, J.; Audouin, L.; Bécares, V.; Barbagallo, M.; Bečvář, F; et al. Measurement and resonance analysis of the ${ }^{33} \mathrm{~S}(n, \alpha)^{30} \mathrm{Si}$ cross section at the CERN $n_{T} O F$ facility in the energy region from 10 to $300 \mathrm{keV}$. Phys. Rev. C 2018, 97, 064603. [CrossRef]

43. Bai, H.; Jiang, H.; Lu, Y.; Cui, Z.; Chen, J.; Zhang, G.; Gledenov, Y.M.; Sedysheva, M.V.; Khuukhenkhuu, G.; Ruan, X.; et al. ${ }^{56,54} \mathrm{Fe}(n, \alpha){ }^{53,51} \mathrm{Cr}$ cross sections in the MeV region. Phys. Rev. C 2019, 99, 024619. [CrossRef]

44. Helgesson, P.; Sjöstrand, H.; Rochman, D. Uncertainty-driven nuclear data evaluation including thermal $(n, \alpha)$ applied to ${ }^{59} \mathrm{Ni}$. Nucl. Data Sheets 2017, 145, 1-24. [CrossRef]

45. Weiß, C.; Griesmayer, E.; Guerrero, C.; Altstadt, S.; Andrzejewski, J.; Audouin, L.; Badurek, G.; Barbagallo, M.; Bécares, V.; Bečvář F.; et al. A new CVD diamond mosaic-detector for $(n, \alpha)$ cross-section measurements at the n_TOF experiment at CERN. Nucl. Instrum. Methods Phys. Res. Sect. A Accel. Spectrometers Detect. Assoc. Equip. 2013, 732, 190-194. [CrossRef]

46. Gyürky, G.; Fülöp, Z.; Käppeler, F.; Kiss, G.G.; Wallner, A. The activation method for cross section measurements in nuclear astrophysics. Eur. Phys. J. A 2019, 55, 41. [CrossRef]

47. Al-Khasawneh, K.; Borris, E.; Bruückner, B.; Eberhardt, K.; Erbacher, P.; Fiebiger, S.; Gernhäauser, R.; Göobel, K.; Heftrich, T.; Kisselbach, T.; et al. NICE-Neutron Induced Charged particle Emission. J. Phys. Conf. Ser. 2020, 1668, 012021. [CrossRef]

48. Hauser, W.; Feshbach, H. The Inelastic Scattering of Neutrons. Phys. Rev. 1952, 87, 366-373. [CrossRef]

49. Moldauer, P.A. Why the Hauser-Feshbach formula works. Phys. Rev. C 1975, 11, 426-436. [CrossRef]

50. Koning, A.; Hilaire, S.; Duijvestijn, M. TALYS1.0. In Proceedings of the International Conference on Nuclear Data for Science and Technology, Nice, France, 22-27 April 2007; EDP Sciences: Les Ulis, France, 2008; pp. 211-214. 
51. Koning, A.; Hilaire, S.; Goriely, S. TALYS-1.8 A Nuclear Reaction Program. User Manual; Nuclear Research and Consultancy Group (NRG): Petten, The Netherlands, 2015.

52. Goriely, S.; Chamel, N.; Pearson, J.M. Skyrme-Hartree-Fock-Bogoliubov Nuclear Mass Formulas: Crossing the $0.6 \mathrm{MeV}$ Accuracy Threshold with Microscopically Deduced Pairing. Phys. Rev. Lett. 2009, 102, 152503. [CrossRef]

53. Audi, G.; Wapstra, A.; Thibault, C. The Ame2003 atomic mass evaluation: (II). Tables, graphs and references. Nucl. Phys. A 2003, 729, 337-676. [CrossRef]

54. Wapstra, A.; Audi, G.; Thibault, C. The Ame2003 atomic mass evaluation: (I). Evaluation of input data, adjustment procedures. Nucl. Phys. A 2003, 729, 129-336. [CrossRef]

55. Newton, J.R.; Iliadis, C.; Champagne, A.E.; Coc, A.; Parpottas, Y.; Ugalde, C. Gamow peak in thermonuclear reactions at high temperatures. Phys. Rev. C 2007, 75, 045801. [CrossRef]

56. Glorius, J.; Langer, C.; Slavkovská, Z.; Bott, L.; Brandau, C.; Brückner, B.; Blaum, K.; Chen, X.; Dababneh, S.; Davinson, T.; et al. Approaching the Gamow Window with Stored Ions: Direct Measurement of ${ }^{124} \mathrm{Xe}(p, \gamma)$ in the ESR Storage Ring. Phys. Rev. Lett. 2019, 122, 092701. [CrossRef] [PubMed]

57. Ciani, G.F.; Csedreki, L.; Rapagnani, D.; Aliotta, M.; Balibrea-Correa, J.; Barile, F.; Bemmerer, D.; Best, A.; Boeltzig, A.; Broggini, C.; et al. Direct Measurement of the ${ }^{13} \mathrm{C}(\alpha, n){ }^{16} \mathrm{O}$ Cross Section into the s-Process Gamow Peak. Phys. Rev. Lett. 2021, 127, 152701. [CrossRef]

58. Rauscher, T. Relevant energy ranges for astrophysical reaction rates. Phys. Rev. C 2010, 81, 045807. [CrossRef]

59. Fallis, J.; Akers, C.; Laird, A.; Simon, A.; Spyrou, A.; Christian, G.; Connolly, D.; Hager, U.; Hutcheon, D.; Lennarz, A.; et al. First measurement in the Gamow window of a reaction for the gamma-process in inverse kinematics: 76Se(alpha,gamma)80Kr. Phys. Lett. B 2020, 807, 135575. [CrossRef]

60. Stoitsov, M.; Moré, J.; Nazarewicz, W.; Pei, J.C.; Sarich, J.; Schunck, N.; Staszczak, A.; Wild, S. Towards the universal nuclear energy density functional. J. Phys. Conf. Ser. 2009, 180, 012082. [CrossRef]

61. Paar, N.; Papakonstantinou, P.; Ponomarev, V.; Wambach, J. Low-energy dipole excitations towards the proton drip-line: Doubly magic 48Ni. Phys. Lett. B 2005, 624, 195-202. [CrossRef]

62. Paar, N. The quest for novel modes of excitation in exotic nuclei. J. Phys. G Nucl. Part. Phys. 2010, 37, 064014. [CrossRef]

63. Khan, E.; Paar, N.; Vretenar, D. Low-energy monopole strength in exotic nickel isotopes. Phys. Rev. C 2011, 84, 051301. [CrossRef]

64. Samana, A.R.; Krmpotić, F.; Paar, N.; Bertulani, C.A. Neutrino and antineutrino charge-exchange reactions on ${ }^{12}$ C. Phys. Rev. C 2011, 83, 024303. [CrossRef]

65. Fantina, A.F.; Khan, E.; Colò, G.; Paar, N.; Vretenar, D. Stellar electron-capture rates on nuclei based on a microscopic Skyrme functional. Phys. Rev. C 2012, 86, 035805. [CrossRef]

66. Paar, N.; Moustakidis, C.C.; Marketin, T.; Vretenar, D.; Lalazissis, G.A. Neutron star structure and collective excitations of finite nuclei. Phys. Rev. C 2014, 90, 011304. [CrossRef]

67. Nikšić, T.; Paar, N.; Vretenar, D.; Ring, P. DIRHB-A relativistic self-consistent mean-field framework for atomic nuclei. Comput. Phys. Commun. 2014, 185, 1808-1821. [CrossRef]

68. Paar, N.; Vretenar, D.; Khan, E.; Colò, G. Exotic modes of excitation in atomic nuclei far from stability. Rep. Prog. Phys. 2007, 70, 691-793. [CrossRef]

69. Gao, Y.; Dobaczewski, J.; Kortelainen, M.; Toivanen, J.; Tarpanov, D. Propagation of uncertainties in the Skyrme energy-densityfunctional model. Phys. Rev. C 2013, 87, 034324. [CrossRef]

70. Roca-Maza, X.; Colò, G.; Sagawa, H. New Skyrme energy density functional for a better description of the Gamow-Teller resonance. Phys. Scr. 2013, T154, 014011. [CrossRef]

71. Washiyama, K.; Bennaceur, K.; Avez, B.; Bender, M.; Heenen, P.H.; Hellemans, V. New parametrization of Skyrme's interaction for regularized multireference energy density functional calculations. Phys. Rev. C 2012, 86, 054309. [CrossRef]

72. Yüksel, E.; Marketin, T.; Paar, N. Optimizing the relativistic energy density functional with nuclear ground state and collective excitation properties. Phys. Rev. C 2019, 99, 034318. [CrossRef]

73. Kohn, W.; Sham, L.J. Self-Consistent Equations Including Exchange and Correlation Effects. Phys. Rev. 1965, 140, A1133-A1138. [CrossRef]

74. Kohn, W. Nobel Lecture: Electronic structure of matter-wave functions and density functionals. Rev. Mod. Phys. 1999, 71, 1253-1266. [CrossRef]

75. Goriely, S.; Hilaire, S.; Koning, A.J. Improved predictions of nuclear reaction rates with the TALYS reaction code for astrophysical applications. A\&A 2008, 487, 767-774. [CrossRef]

76. Avrigeanu, V.; Avrigeanu, M.; Mănăilescu, C. Further explorations of the $\alpha$-particle optical model potential at low energies for the mass range $A \approx 45-209$. Phys. Rev. C 2014, 90, 044612. [CrossRef]

77. Koning, A.; Hilaire, S.; Goriely, S. New Edition-24 December 2019. Available online: https://tendl.web.psi.ch/tendl_2019/talys. html (accessed on 28 November 2021).

78. Moldauer, P. Statistics and the average cross section. Nucl. Phys. A 1980, 344, 185-195. [CrossRef]

79. Krane, K. Introductory Nuclear Physics, 2nd ed.; John Willey and Sons: New York, NY, USA, 1988.

80. Koehler, P.E.; Graff, S.M. ${ }^{17} \mathrm{O}(\mathrm{n}, \alpha){ }^{14} \mathrm{C}$ cross section from $25 \mathrm{meV}$ to approximately $1 \mathrm{MeV}$. Phys. Rev. C 1991, 44, 2788-2793. [CrossRef] [PubMed] 
81. Koehler, P.; Harvey, J.; Hill, N. Two detectors for $(n, p)$ and $(n, \alpha)$ measurements at white neutron sources. Nucl. Instrum. Methods Phys. Res. Sect. A Accel. Spectrometers Detect. Assoc. Equip. 1995, 361, 270-276. [CrossRef]

82. Wagemans, C.; Weigmann, H.; Barthelemy, R. Measurement and resonance analysis of the ${ }^{33} \mathrm{~S}(n, \alpha)$ cross section. Nucl. Phys. A 1987, 469, 497-506. [CrossRef]

83. Koning, A.; Rochman, D.; Sublet, J.C.; Dzysiuk, N.; Fleming, M.; van der Marck, S. TENDL: Complete Nuclear Data Library for Innovative Nuclear Science and Technology. Nucl. Data Sheets 2019, 155, 1-55. [CrossRef]

84. Brown, D.; Chadwick, M.; Capote, R.; Kahler, A.; Trkov, A.; Herman, M.; Sonzogni, A.; Danon, Y.; Carlson, A.; Dunn, M.; et al. ENDF/B-VIII.0: The 8th Major Release of the Nuclear Reaction Data Library with CIELO-project Cross Sections, New Standards and Thermal Scattering Data. Nucl. Data Sheets 2018, 148, 1-142. [CrossRef]

85. Blokhin, A.I.; Gai, E.V.; Ignatyuk, A.V.; Koba, I.I.; Manokhin, V.N.; Pronyaev, V.N. New version of neutron evaluated data library BROND-3.1. Yad. Reak. Konst. 2016, 2, 62.

86. Oginni, B.M.; Iliadis, C.; Champagne, A.E. Theoretical evaluation of the reaction rates for ${ }^{26} \mathrm{Al}(n, p){ }^{26} \mathrm{Mg}$ and ${ }^{26} \mathrm{Al}(n, \alpha){ }^{23} \mathrm{Na}$. Phys. Rev. C 2011, 83, 025802. [CrossRef]

87. Herman, M.; Capote, R.; Carlson, B.; Oblozinsky, P.; Sin, M.; Trkov, A.; Wienke, H.; Zerkin, V. EMPIRE: Nuclear Reaction Model Code System for Data Evaluation. Nucl. Data Sheets 2007, 108, 2655-2715. [CrossRef] 